\title{
Janus Study Data Repository
}

National Cancer Institute

\section{Source}

National Cancer Institute. Janus Study Data Repository. NCI Thesaurus. Code C142595.

An electronic repository within the US Food and Drug Administration containing subject-

level clinical and nonclinical study data submitted to the FDA as part of a regulatory submission. 